University of Windsor

Scholarship at UWindsor

$1-22-2018$

\title{
Solution Deposition of Conformal Gold Coatings on Knitted Fabric for E-Textiles and Electroluminescent Clothing
}

Tricia Carmichael

Yunyun Wu

University of Windsor

Sara S. Mechael

University of Windsor

Yiting Chen

University of Windsor

Follow this and additional works at: https://scholar.uwindsor.ca/chemistrybiochemistrypub

Part of the Materials Chemistry Commons, and the Materials Science and Engineering Commons

\section{Recommended Citation}

Carmichael, Tricia; Wu, Yunyun; Mechael, Sara S.; and Chen, Yiting. (2018). Solution Deposition of Conformal Gold Coatings on Knitted Fabric for E-Textiles and Electroluminescent Clothing. Advanced Materials Technologies, 3 (3), 1700292.

https://scholar.uwindsor.ca/chemistrybiochemistrypub/94

This Article is brought to you for free and open access by the Department of Chemistry and Biochemistry at Scholarship at UWindsor. It has been accepted for inclusion in Chemistry and Biochemistry Publications by an authorized administrator of Scholarship at UWindsor. For more information, please contact scholarship@uwindsor.ca. 


\title{
WILEY-VCH
}

DOI: $10.1002 /(($ please add manuscript number $))$

Article type: Communication

\section{Solution Deposition of Conformal Gold Coatings on Knitted Fabric for E-Textiles and Electroluminescent Clothing}

Yunyun Wu, Sara S. Mechael, Yiting Chen, and Tricia Breen Carmichael*

Y. Wu, S. S. Mechael, Y. Chen, Prof. T. B. Carmichael

Department of Chemistry and Biochemistry, University of Windsor, Windsor, Ontario, N9B 3P4 Canada

E-mail: tbcarmic@uwindsor.ca

Keywords: e-textile, printed electronics, stretchable conductors, electroluminescent devices, wearable electronics

\begin{abstract}
The vision for wearable electronics involves creating an imperceptible boundary between humans and devices. Integrating electronic devices into clothing represents an important path to this vision; however, combining conductive materials with textiles is challenging due to the porous structure of knitted textiles. Stretchability depends on maintaining the void structure between the yarns of the fabric; filling these voids with conductive materials stiffens the textile and can lead to detrimental cracking. The authors demonstrate the solution-based metallization of a knitted textile that conformally coats individual fibers with gold, leaving the void structure intact. The resulting gold-coated textile is highly conductive, with a sheet resistance of $1.07 \Omega \mathrm{sq}^{-1}$ in the course direction. The resistance decreases by $80 \%$ when the fabric is stretched to $15 \%$ strain, and remains at this value to $160 \%$ strain. This outstanding combination of stretchability and conductivity is accompanied by durability to wearing, sweating, and washing. Low-cost screen printing of a wax resist is demonstrated to produce patterned gold textiles suitable for electrically connecting discrete devices in clothing. The fabrication of electroluminescent fabric by depositing layers of device materials onto the
\end{abstract}




\section{WILEY-VCH}

gold-coated textile is furthermore demonstrated, intimately merging device functionality with textiles for imperceptible wearable devices.

Textile-based wearable electronics (e-textiles) will unlock a future with a redefined human-computer interaction, in which interacting with devices is as simple as getting dressed. For truly wearable electronics, it is essential to integrate electrical functionality while maintaining the soft, stretchable properties of the textile, and the look and feel the end-user expects. The rapidly developing field of skin-mounted wearable electronics has successfully addressed the fundamental mismatch between conventional hard electronics and soft substrates by developing an impressive range of soft devices on elastomers that can be laminated on the skin, including biosensors, ${ }^{[1]}$ displays, ${ }^{[2]}$ and power sources. ${ }^{[3]}$ Integrating such sophisticated function into textiles, however, is vastly different from fabrication on the flat surfaces of elastomers due to the porous, 3D structure of knitted or woven fabrics. This difference presents special challenges to the field of e-textiles, distinguishing it conceptually from elastomer-based stretchable electronics. A fundamental step toward sophisticated etextiles involves endowing textiles with electrical conductivity, which forms the basis of two design concepts: The first concept uses conductive wiring integrated in the textile to form a power bus and data network to connect multiple, separate devices, effectively decoupling device fabrication from the textile and enabling the distribution of high-performance devices over different locations in clothing. ${ }^{[4]}$ The second concept seeks more intimate merging of devices with textiles by using a conductive textile as a base on which to build up layers of functional materials to form highly integrated, imperceptible devices. ${ }^{[5]}$ Both approaches require highly conductive textiles with stable conductivity under strain for consistent performance, as well as stretchability and flexibility. 


\section{WILEY-VCH}

Knitted or woven textiles present a unique challenge owing to their complex, porous 3D structure, which consists of an interconnected network of fiber bundles (yarns) separated by a network of voids. These voids are key to the flexibility and stretchability of the fabric by providing space for the yarns to move and slip in response to stress. Creating conductive pathways by filling these voids with conductive inks or pastes, such as conductive polymers, ${ }^{[6]}$ or composites of polymers with graphene, ${ }^{[7]}$ carbon nanotubes,${ }^{[8]}$ silver nanoparticles, ${ }^{[9]}$ or silver flakes, ${ }^{[10]}$ forms a conductive composite; however, this approach restricts the movement of yarns and stiffens the textile. Such composites are also vulnerable to cracking under strain. ${ }^{[7]}$ Weaving, ${ }^{[11]}$ knitting $^{[12]}$ or embroidering ${ }^{[13]}$ metal wires or conductive threads into the fabric is one way to add conductivity while maintaining the void network, although this approach can be laborious, and breakage of metal wires during the manufacturing process is a concern. ${ }^{[14]}$ Another tactic has been to develop methods that coat only the individual yarns or fibers of fabric with conductive materials, which leaves the void network unchanged to retain stretchability. For example, Jin et al. developed specialized conductive ink formulations to control the permeation of the ink in the textile and maintain the voids. $^{[15]}$ Other approaches use in situ polymerization of conductive polymers ${ }^{[16]}$ in the textile or carbonization ${ }^{[17]}$ of textiles through thermal treatment; however, the former typically results in low conductivity, and the latter requires cellulose-based textiles that convert to graphite-like materials at very high temperatures $\left(\sim 1000^{\circ} \mathrm{C}\right)$.

Depositing thin metal coatings on textiles is a promising approach to afford high conductivity while maintaining the void structure necessary for stretchability. Physical vapor deposition (PVD) of metals is a standard method used to prepare metal films; however, its line-of-sight nature is incompatible with the 3D structures of woven or knitted textiles (vide infra). Although PVD methods are suitable for nonwoven fabrics, which are made by binding fibers together in a planar sheet or web, such fabrics are not stretchable. ${ }^{[18]}$ Additional planarization layers, such as polyurethanes, deposited on the fabric can make PVD methods 


\section{WILEY-VCH}

applicable to stretchable knitted or woven fabrics, but this approach fills the void structure and restricts the stretchability. ${ }^{[19]}$ Electroless metal deposition (ELD) is a solution-based alternative to PVD with an important advantage for knitted and woven fabrics: The ELD plating solution can permeate the fabric structure to deposit metal on individual fibers, leaving the void structure intact. ${ }^{[20]}$ Metals deposited on woven cotton or nylon textiles using ELD, such as copper, ${ }^{[20 \mathrm{a}, 20 \mathrm{~b}]}$ silver, ${ }^{[20 \mathrm{c}]}$ and nickel, ${ }^{[20 \mathrm{~d}-\mathrm{h}]}$ exhibit low sheet resistances $(<1 \Omega / \mathrm{sq})$, but the stretchability is limited by the structure of the woven fabric, which consists of interlacing yarns at right angles that restrict stretching to the diagonal direction. Furthermore, the metals demonstrated in these studies are problematic for wearables due to the propensity of silver and copper to oxidize or corrode, ${ }^{[21]}$ particularly in contact with salt solutions like human sweat, and the prevalence of contact dermatitis caused by nickel metal. ${ }^{[22]}$

Here, we describe the solution deposition of patterned gold coatings on knitted fabric that are conductive, stretchable, wearable, and washable. We chose gold as the metal because it is biocompatible, chemically inert, and not easily oxidized. ${ }^{[23]}$ Our simple, low-cost method uses electroless nickel immersion gold (ENIG) plating, a solution-based technique commonly employed in printed circuit board fabrication. ${ }^{[24]}$ The ENIG process deposits a gold coating over the individual textile fibers, producing a highly conductive textile that retains the softness and stretchability of the knitted fabric. We demonstrate screen printing of a wax resist to pattern the deposition of gold on the fabric, and furthermore employ gold-coated textiles to fabricate stretchable and wearable electroluminescent textiles.

We chose a weft-knitted polyester fabric with a water-resistant polyurethane backing as our substrate. Weft-knitted fabrics consist of wale (column) loops and course (row) loops interlinked with each other (Figure 1a, b). The polyester fiber of the fabric is not stretchable; rather, the fabric is stretchable due to the serpentine loops in the knitted structure. Stretching along the course direction straightens the curved serpentine yarns, increasing the wale width and decreasing the course height. Stretching along the wale direction increases the course 


\section{WILEY-VCH}

height and decreases the wale width. Using PVD to deposit gold on the weft-knitted textile highlights the complexity of the interlinked structure and, consequently, its incompatibility with the line-of-sight PVD process. Overlying yarns at the interlinked course and wale junctions block gold deposition on the underlying yarns, producing a discontinuous gold coating with broken conductive pathways along the course (Figure 1c, d) and the wale (Figure 1e, f) directions.

We used the solution-based ENIG method to uniformly coat the fibers of the weftknitted textile with gold and create conductive pathways throughout the textile structure. The ENIG process consists of two solution-based plating steps: electroless deposition of nickel followed by an immersion gold process. The ELD nickel process involves first activating the textile by adsorbing a catalytic species onto its surface. We used a palladium-tin colloidal catalyst, which consists of a palladium-rich core protected from oxidation by a hydrolyzed $\mathrm{Sn}^{2+} / \mathrm{Sn}^{4+}$ shell, and an associated chloride layer that gives the colloids a negatively charged surface. ${ }^{[25]} \mathrm{Pd} / \mathrm{Sn}$ colloids can be bound electrostatically to positively-charged surfaces through a 3-aminopropyltriethoxysilane (APTES) linker, ${ }^{[26]}$ which we deposited onto the fabric from solution after a plasma oxidation step. Immersing the resulting aminefunctionalized textile in the acidic $\mathrm{Pd} / \mathrm{Sn}$ solution protonates the amino groups of the bound APTES; the resulting positively-charged ammonium groups electrostatically bind the $\mathrm{Pd} / \mathrm{Sn}$ colloids. Subsequent etching in aqueous $\mathrm{HCl}$ removes the $\mathrm{Sn}$ shell to expose the Pd core, which initiates ELD by reducing $\mathrm{Ni}^{2+}$ ions in the ELD plating solution to nickel metal on the surface of the textile. Nickel deposition is autocatalytic thereafter due to the dimethylamine borane reducing agent in the nickel ELD solution. In the second ENIG plating step, immersing the nickel-coated textile in a solution of potassium gold cyanide deposits a gold film by galvanic displacement. $\mathrm{Ni}$ atoms in the film reduce $\mathrm{Au}^{+}$ions from solution, releasing $\mathrm{Ni}^{2+}$ ions into the solution and depositing a gold metal film on the surface through molecular exchange. ${ }^{[24]}$ Throughout the ENIG process, floating the polyester front face of the fabric on 


\section{WILEY-VCH}

the surface of the plating solutions prevents metallization of the polyurethane-coated backside (Figure S1), which can facilitate integration into wearable electronics by separating the conductive layer from the body and preventing possible shorts caused by sweat.

The aqueous solutions used in the ENIG process permeate the fabric to coat the individual fibers with metal, including in the junctions of wale and course yarn, rendering the fabric electrically conductive (Figure 2a-c). SEM images show the smooth, conformal gold coating on the individual fibers, which appear similar to the pristine fabric due to the uniformity of the coating (Figure 2d, e, Figure S2). Cross-sectional SEM shows that each fiber consists of a core-shell structure, with each polyester fiber core coated with a $\sim 80-\mathrm{nm}$ thick gold shell (Figure 2f). Analysis of the films using X-ray diffraction and energydispersive X-ray spectroscopy detected gold, but not nickel, in the gold-coated textile (Figure S3). The gold-coated textile is highly conductive, with an average sheet resistance $\left(R_{s}\right)$ in the course direction $(1.07 \pm 0.23 \Omega / \mathrm{sq})$ comparable to that of a flat 80 -nm-thick film of gold deposited by PVD on a glass substrate $(0.9 \pm 0.1 \Omega / s q) . R_{s}$ in the wale direction is slightly higher $(3.33 \pm 1.49 \Omega /$ sq $)$, which can be attributed to additional interfiber contact resistance between loops in the wale direction.

Facile permeation of ENIG solutions into the porous structure of the weft-knitted textile brings the benefit of coating individual fibers with metal; however, this porosity makes it a challenge to pattern the deposition of metal on the textile to create defined conductive features. We used a hydrophobic wax resist to pattern ENIG deposition on the weft-knitted textile (Figure 3a). Molten wax printing is commonly used to fabricate hydrophobic barriers in applications involving porous substrates, from conventional batik printing to pattern the deposition of dye onto textiles, to defining microfluidic channels in paper-based analytical devices. ${ }^{[27]}$ We used cold wax medium, a beeswax-based paste that is soft, viscous, and suitable for screen printing. Applying the cold wax medium though a stencil mask to plasma- 


\section{WILEY-VCH}

oxidized fabric forms a patterned hydrophobic barrier via the penetration of cold wax medium into the fabric (Figure S4). The hydrophobic barrier blocks the adsorption of $\mathrm{Pd} / \mathrm{Sn}$ colloids from solution, thereby rendering these areas of the fabric inactive to ENIG. We then removed the wax resist by dissolution in hexane to leave a pattern of $\mathrm{Pd} / \mathrm{Sn}$ catalyst on the fabric.

Subsequent ENIG processing deposits metal only in these regions to render them conductive, whereas the regions that were masked with the wax resist are non-conductive. We studied the resolution and fidelity of the screen printing process using stencil masks designed to produce two types of features: gold lines (Figure 3b-e) and linear gaps in gold films (Figure $3 \mathrm{f}-\mathrm{i}$ ) with widths from $100 \mu \mathrm{m}$ to $1000 \mu \mathrm{m}$. Plots of the measured feature width as a function of the nominal feature width given by the stencil mask (Figure S5) yields a linear relationship with slope $=1$ for both feature types. Two factors can potentially influence the resolution: Lateral wicking of the cold wax medium into the fabric will cause narrowing of plated metal lines and widening of linear gaps compared to the nominal widths. On the other hand, the metal growth mechanism may cause widening of plated metal lines and narrowing of linear gaps due to accumulation of metal in the $\mathrm{x}-\mathrm{y}$ plane when longer plating times are used. Nonetheless, we found that our printing and plating conditions produced line and gap features that showed excellent fidelity to feature widths as small as $300 \mu \mathrm{m}$, with lateral wicking of the cold wax medium through the fabric preventing the fabrication of smaller lines and gaps.

The gold-coated fabric possesses mechanical and electrical characteristics that make it suitable for applications in wearable electronics. Since ENIG deposits gold conformally on the surfaces of the fabric fibers rather than depositing material in between the yarns, the movement of the loops is not restricted and the stretchability is maintained. The gold-coated fabric largely retains the original mechanical properties of the fabric, although tensile testing before and after metallization showed a slight decrease in tensile strength and breaking load (Figure S6). This change may be due to the increased interfiber friction caused by the metal coating, which may limit the gliding action of yarns. ${ }^{[28]}$ The gold-coated fabric also retains its 


\section{WILEY-VCH}

conductivity with stretching, a key attribute for using conductive fabric in clothing. We stretched the gold-coated fabric in the course and wale direction and measured the change in resistance with strain (Figure $4 \mathrm{a}$ ). In both directions, stretching to $15 \%$ strain rapidly decreases the resistance to $\sim 15 \%$ of the initial value. This decrease is due to an increase in the contact pressure between adjacent conductive yarns that occurs under tensile strain, which lowers the interfiber contact resistance. ${ }^{[29]}$ The maximum effect of this contact pressure occurs at $15 \%$ strain, beyond which the resistance remains consistent even as the fabric is elongated to $80 \%$ strain in the wale direction and $160 \%$ strain in the course direction. At this point, the loops of the fabric are interlocked and the fabric can be stretched no further. The difference in the maximum strain in the course and wale directions is due to the anisotropic structure of the textile, which has a larger course height compared to the wale width, causing the interlock to occur at higher elongations in the course direction. The excellent conductivity and stretching performance is likely facilitated by the robust adhesion between the metal and the fabric, which prevents metal delamination and cracking that typically lead to resistance increases and termination of conductivity in metal-on-polymer structures. ${ }^{[30]}$ Mechanical adhesion testing of the gold coating using the tape test showed no visible gold on the tape (Figure S7a, b), and SEM images of the gold-coated textile at $20 \%$ strain show no evidence of metal cracking or peeling (Figure S8). The APTES molecular layer deposited on the fabric surface is a key contributor to the robust adhesion: Coating the fabric using an ENIG process that omitted the APTES deposition resulted in a gold film poorly adhered to the fabric, and failure of the tape test (Figure S7c, d).

We further investigated the durability of the gold-coated fabric in wearable electronics by incorporating it into clothing. We sewed $1.5 \times 3 \mathrm{~cm}^{2}$ patches of gold-coated textile onto the knee sections of a pair of exercise pants (Figure $4 \mathrm{~b}$ ), and then subjected them to repeated knee bending ( $\sim 40 \%$ strain) by wearing the pants for a $5-\mathrm{km}$ running session $(\sim 3500$ cycles $)$. The resistance of the gold-coated fabric increased $<10 \%$ after running for $5 \mathrm{~km}$ (Table S1). 


\section{WILEY-VCH}

We conducted additional stretch-release experiments by cycling the gold-coated fabric between 0 and $40 \%$ strain. We measured the resistance at each $40 \%$ elongation and again after each release, allowing the gold-coated textile to relax for 2 minutes before measurement (Figure S9). The data show that, as expected, the resistance of the gold-coated textile decreases to $\sim 15 \%$ of the initial value at each $40 \%$ strain cycle. At each release cycle, however, the resistance does not recover to its initial value, instead returning to $\sim 50 \%$ of the initial value. This recovery is consistent with low resilience of the knitted polyester fabric, which does not incorporate elastic fibers into the knitted structure that are typically used in other fabrics to enable rapid recovery to the original shape. In knitted fabrics that lack elastic fibers, stretching straightens the serpentine polyester loops, and recovery requires additional time for the loops to bend back to their original shape. Stretching the gold-coated textile to $40 \%$ strain and then allowing the fabric to relax for $12 \mathrm{~h}$ enables the recovery of the initial resistance, with a normalized resistance of $1.01 \pm 0.14$.

Along with durability to repeated movement, wearable conductors must also be hardy enough to tolerate mild abrasion and laundry cycles. Rapidly rubbing the surface of the goldcoated textile with a gloved finger for 5 minutes caused minimal changes to the sheet resistance, with normalized values of $1.03 \pm 0.17$ in the course direction and $0.83 \pm 0.28$ in the wale direction after the test. We simulated a laundry cycle by stirring the gold-coated textile in an aqueous solution of laundry detergent for $1 \mathrm{~h}$, followed by rinsing with water and drying in a $60^{\circ}$ oven overnight. The sheet resistance of the gold-coated textile varied minimally throughout 10 simulated laundry cycles (Figure 4c). Wearable electronics worn near human skin must also be tolerant to sweating. We immersed the gold-coated textile in a salt solution to simulate exposure to sweat, and then subjected it a laundry cycle. There was minimal change in resistance after each step: After sweat exposure, the normalized change in sheet resistance in the course and wale directions was $0.99 \pm 0.31$ and $0.89 \pm 0.09$, 


\section{WILEY-VCH}

respectively; after the subsequent laundry cycle, the values in the course and wale directions were $1.01 \pm 0.11$ and $0.81 \pm 0.20$, respectively.

We used the gold-coated textile to fabricate electroluminescent fabric, demonstrating the ultimate integration of device functionality with textiles. The electroluminescent fabric uses the gold-coated textile as the bottom electrode in an alternating current electroluminescent (ACEL) device (Figure 5a). ACEL devices consist of a light-emitting phosphor material deposited between two electrodes; stretchable ACELs have previously been fabricated by blending $\mathrm{ZnS}: \mathrm{Cu}$ phosphor microparticles with the elastomer polydimethylsiloxane (PDMS), and using stretchable PDMS-based electrodes. ${ }^{[31]} \mathrm{We}$ fabricated a stretchable ACEL on the gold-coated textile by first depositing a stretchable dielectric layer consisting of a blend of barium titanate and PDMS onto the gold-coated textile to smooth the uneven surface of the textile and prevent breakdown of the phosphor under the high field strengths of the device. We deposited a film of $\mathrm{ZnS}: \mathrm{Cu}$ blended with PDMS, and then completed the device by depositing a film of the conductive polymer PEDOT:PSS plasticized with the surfactant Triton $\mathrm{X}-100^{[32]}$ as the transparent electrode. Operation at 165 $\mathrm{V} \mathrm{AC}$ and a frequency of $37 \mathrm{kHz}$ produced uniform blue emission from the electroluminescent fabric. The device functionality persisted without degradation to $40 \%$ strain (Figure 5b). Stretching beyond $40 \%$ strain caused device failure due to a loss of conductivity of the PEDOT:PSS electrode. The stretchability of the electroluminescent fabric could be improved by using a transparent electrode with high conductivity and stretchability. Nonetheless, electroluminescent functionality from 0 to $40 \%$ strain is a workable range ${ }^{[33]}$ for applications such as high-visibility safety apparel, which is worn to alert drivers to the presence of a worker, pedestrian, or cyclist in low light or poor visibility conditions.

Retroreflective materials used today in safety apparel require an external source of light for visibility, such as car headlights. We integrated the electroluminescent fabric into clothing to demonstrate self-illuminated safety apparel (Figure 5c, d), which provides consistent visibility 


\section{WILEY-VCH}

regardless of external conditions to enhance the safety of the wearer. A comparison of retroreflective and electroluminescent fabric strips in clothing photographed in light and dark environments shows the superior brightness and visibility of the electroluminescent fabric (Figure 5c, d).

In conclusion, we believe that our simple and scalable ENIG method provides conductive textiles with properties that fit the needs of future wearable electronics. The permeation of ENIG solutions into the porous structure of the weft-knitted polyester textile enables the deposition of a uniform, conformal gold film only on the fabric fibers, preserving the void structure and stretchability of the textile. The adhesion of the gold coating makes this e-textile durable to wearing, sweating, and washing. The stable conductivity of the gold coating to high strains combined with the low-cost screen printing of a wax resist enables the formation of patterned gold textiles that will be useful to electrically connect a network of devices on the body, delivering power and enabling communication. The gold-coated textiles may also be useful in wearable strain sensors over the $0-15 \%$ strain range where the resistance decreases with strain. ${ }^{[34]}$ The electroluminescent fabric demonstrated herein demonstrates the merging of devices with fabrics, and represents the first steps toward the fabrication of imperceptible, wearable displays. We are currently investigating the use of the ENIG process to metallize other fabrics, such as cotton or nylon, as well as fabrics that incorporate elastic fibers to enable high stretchability and conformability to the human body with high resilience.

\section{Experimental Section}

All chemicals were purchased commercially and used as received.

ENIG Procedure: Polyester fabric with ca. $200-\mu \mathrm{m}$-diameter threads and polyurethane backing was sonicated in deionized water and isopropyl alcohol for $15 \mathrm{~min}$ each, and then exposed to air plasma (15 psi) for $6 \mathrm{~min}$. The patterned fabric was floated on the surface of a 


\section{WILEY-VCH}

$1 \% \mathrm{v} / \mathrm{v}$ solution of APTES in deionized water for $10 \mathrm{~min}$, a $\mathrm{Pd} / \mathrm{Sn}$ solution (prepared from Cataposit 44 and Cataprep 404 (Shipley) as directed by manufacturer) for $3 \mathrm{~min}$, and aqueous $6 \mathrm{M} \mathrm{HCl}$ for $3 \mathrm{~min}$. Samples were rinsed with water in between steps. The fabric was then metallized in nickel ELD bath $\left(21.0 \mathrm{~g} \mathrm{~L}^{-1} \mathrm{NiSO}_{4} \cdot 6 \mathrm{H}_{2} \mathrm{O}, 37.2 \mathrm{~g} \mathrm{~L}^{-1} \mathrm{Na}_{4} \mathrm{P}_{2} \mathrm{O}_{7} \cdot 10 \mathrm{H}_{2} \mathrm{O}\right.$ and $4.1 \mathrm{~g}$ $\mathrm{L}^{-1}$ dimethylamine borane in water) for $5 \mathrm{~min}$ with sonication. After rinsing with water, the Ni-coated fabric was immersed in Au ELD bath (Gobright TAM-55, Uyemura) for 30 min. Patterning of Polyester Fabric: The fabric was sonicated in deionized water and isopropyl alcohol for $15 \mathrm{~min}$ each, and then exposed to air plasma (15 psi) for $6 \mathrm{~min}$. A $0.15 \mathrm{~mm}$ stainless steel stencil with the desired pattern was placed on top of the fabric, cold wax medium (Gamblin) was applied to cover the metal stencil, and then a glass microscope slide was used as a squeegee to remove the excess. The ENIG procedure was then performed. Washing Durability: Gold-coated textiles were soaked in $1 \mathrm{wt} \%$ solution of Tide Original Liquid Laundry Detergent in DI water with stirring for $1 \mathrm{~h}$, followed by rinsing with water and drying in a $60^{\circ} \mathrm{C}$ oven overnight. The sheet resistance was measured before and after each washing cycle using the four-point probe method.

Sweating Durability The gold-coated textiles were immersed in salt solution $(0.9 \mathrm{~g} / \mathrm{L} \mathrm{NaCl}$ and $0.2 \mathrm{~g} / \mathrm{L} \mathrm{KCl}$ ) for 3 hours and then dried on a hot plate at $37{ }^{\circ} \mathrm{C}$ for 2 days. The samples were then subjected to the washing procedure. Sheet resistance was measured after samples were dried.

Electroluminescent Device Fabrication: The dielectric layer was formed by mixing PDMS prepolymer (Sylgard 184, Dow Corning, prepared using 10:1 ratio) with $\mathrm{BaTiO}_{3}$ powder in a 1:1 (w:w) ratio, spin coating on the metallized fabric at $1000 \mathrm{rpm}$ for $1 \mathrm{~min}$, and curing in a $60{ }^{\circ} \mathrm{C}$ oven overnight. The emissive layer was then formed by mixing $\mathrm{ZnS}: \mathrm{Cu}$ phosphor powder (KPT) with PDMS prepolymer in 1:1 (w:w) ratio, spin-coating on the dielectric layer at $500 \mathrm{rpm}$ for $1 \mathrm{~min}$, and curing in a $60^{\circ} \mathrm{C}$ oven overnight. The emissive layer was treated with air plasma for $1 \mathrm{~min}$, and then a PEDOT:PSS aqueous dispersion (Clevious PH1000, 


\section{WILEY-VCH}

Heraeus) with $2 \mathrm{wt} \%$ Triton X-100 was spin coated on the emissive layer at $2000 \mathrm{rpm}$ for 1 min and then annealed at $100{ }^{\circ} \mathrm{C}$ for 30 minutes.

\section{Supporting Information}

Supporting Information is available from the Wiley Online Library or from the author.

\section{Acknowledgements}

This research was supported by the National Sciences and Engineering Research Council of Canada (NSERC) through Discovery grant 312167-2012-RGPIN. Y. W. was supported by a MITACS Globalink Graduate Scholarship. We thank R. S. Carmichael and C. Noade for photography, K. Schlingman for helpful discussions, and K. Breen for needlework.

Received: ((will be filled in by the editorial staff))

Revised: ((will be filled in by the editorial staff)) Published online: ((will be filled in by the editorial staff))

\section{References}

[1] H. Kudo, T. Sawada, E. Kazawa, H. Yoshida, Y. Iwasaki, K. Mitsubayashi, Biosens. Bioelectron. 2006, 22, 558.

[2] T. Sekitani, H. Nakajima, H. Maeda, T. Fukushima, T. Aida, K. Hata, T. Someya, Nat. Mater. 2009, 8, 494.

[3] S. Xu, Y. Zhang, J. Cho, J. Lee, X. Huang, L. Jia, J. A. Fan, Y. Su, J. Su, H. Zhang, H. Cheng, B. Lu, C. Yu, C. Chuang, T. Kim, T. Song, K. Shigeta, S. Kang, C. Dagdeviren, I. Petrov, P. V. Braun, Y. Huang, U. Paik, J. A. Rogers, Nat. Commun. 2013, 4, 1543. a) M. M. Gorlick, in Proc. of the $3^{\text {rd }}$ Intern. Symp. on Wearable Computers, IEEE

Computer Society, Los Alamitos, USA 1999, pp. 114-121; b) L. M. Castano, A. B. Flatau, Smart Mater. Struct. 2014, 23, 53001.

[5] K. Cherenack, L. van Pieterson, J. Appl. Phys. 2012, 112, 91301.

[6] M. R. Moraes, A. C. Alves, F. Toptan, M. S. Martins, E. M. F. Vieira, A. J. Paleo, A. P. Souto, W. L. F. Santos, M. F. Esteves, A. Zille, J. Mater. Chem. C 2017, 5, 3807. 


\section{WILEY-VCH}

[7] P. Cataldi, L. Ceseracciu, A. Athanassiou, I. S. Bayer, ACS Appl. Mater. Interfaces 2017, 9, 13825.

[8] S. Wang, S. Xuan, M. Liu, L. Bai, S. Zhang, M. Sang, W. Jiang, X. Gong, Soft Matter 2017, 13, 2483.

[9] I. Kazani, C. Hertleer, D. G. Mey, A. Schwarz, Fibres Text. East. Eur. 2012, 90, 57.

[10] N. Matsuhisa, M. Kaltenbrunner, T. Yokota, H. Jinno, K. Kuribara, T. Sekitani, T. Someya, Nat. Commun. 2015, 6, 7461.

[11] J. D. Ryan, D. Mengistie, R. Gabrielsson, A. Lund, C. Müller, ACS Appl. Mater. Interfaces 2017, 9, 9045.

[12] S. Seyedin, J. M. Razal, P. C. Innis, A. Jeiranikhameneh, S. Beirne, G. G. Wallace, ACS Appl. Mater. Interfaces 2015, 7, 21150.

[13] E. R. Post, M. Orth, P. R. Russo, IBM Syst. J. 2000, 39, 840.

[14] M. Orth, MRS Proceedings 2002, 736, 37.

[15] H. Jin, N. Matsuhisa, S. Lee, M. Abbas, T. Yokota, T. Someya, Adv. Mater. 2017, 29, 1605848.

[16] a) Z. Zhang, X. Shi, H. Lou, Y. Xu, J. Zhang, Y. Li, X. Cheng, H. Peng, J. Mater.

Chem. C 2017, 5, 4139; b) J. Wu, D. Zhou, C. O. Too, G. G. Wallace, Synth. Met. 2005, 155, 698.

[17] a) M. Zhang, C. Wang, X. Liang, Z. Yin, K. Xia, H. Wang, M. Jian, Y. Zhang, Adv. Electron. Mater. 2017, 3, 1700193; b) C. Wang, X. Li, E. Gao, M. Jian, K. Xia, Q. Wang, Z. Xu, T. Ren, Y. Zhang, Adv. Mater. 2016, 28, 6640; c) C. Wang, M. Zhang, K. Xia, X. Gong, H. Wang, Z. Yin, B. Guan, Y. Zhang, ACS Appl. Mater. Interfaces 2017, 9, 13331.

[18] Q. Wei, L. Yu, N. Wu, S. Hong, J. Ind. Text. 2008, 37, 275. 


\section{WILEY-VCH}

[19] a) W. Kim, S. Kwon, Y. Han, E. Kim, K. Choi, S. H. Kang, B. C. Park, Adv.

Electron. Mater. 2016, 2, 1600220; b) L. N. Silva, L. M. Gonçalves, H. Carvalho, J. Mater.

Sci. Mater. Electron. 2013, 24, 635.

[20] a) H. Zhao, L. Hou, J. X. Wu, Y. X. Lu, J. Mater. Chem. C 2016, 4, 7156; b) S. Liu, M. Hu, J. Yang, J. Mater. Chem. C 2016, 4, 1320; c) H. Zhen, K. Li, C. Chen, Y. Yu, Z. Zheng, J. Mater. Chem. A 2017, 5, 782; d) F. Haghdoost, V. Mottaghitalab, A. Haghi, Sensor Rev. 2015, 35, 20; e) Y. Yang, Q. Huang, L. Niu, D. Wang, C. Yan, Y. She, Z. Zheng, Adv. Mater. 2017, 29, 1606679; f) X. Pu, M. Liu, L. Li, S. Han, X. Li, C. Jiang, C. Du, J. Luo, W. Hu, Z. Wang, Adv. Energy Mater. 2016, 6, 1601254; g) R. H. Guo, S. X. Jiang, Y. D. Zheng, J. W. Lan, J. Appl. Polym. Sci. 2013, 127, 4186; h) L. Liu, Y. Yu, C. Yan, K. Li, Z. Zheng, Nat. Commun. 2015, 6, 7260; i) Y. You, C. Yan, Z. Zheng, Adv. Mater. 2014, 26, 5508.

[21] S. P. Sharma, J. Electrochem. Soc. 1978, 125, 2005.

[22] J. P. Thyssen, T. Menné, Chem. Res. Toxicol. 2010, 23, 309.

[23] Encyclopedia of World Environment History Vol. 3 (Eds: S. Krech, J. R. McNeil, C. Merchant), Routledge, London, UK 2004.

[24] H. Liu, N. Li, S. Bi, D. Li, J. Electrochem. Soc. 2007, 154.

[25] Electroless Plating: Fundamentals and Applications (Eds: G. O. Mallory, J. B.

Hajdum), American Electroplaters and Surface Finisher Society, Orlando, FL 1990.

[26] M. S. Miller, G. J. E. Davidson, B. J. Sahli, C. M. Mailloux, T. B. Carmichael, $A d v$. Mater. 2008, 20, 59.

[27] E. Carrilho, A. W. Martinez, G. M. Whitesides, Anal. Chem. 2009, 81, 7091.

[28] F. Mohtaram, V. Mottaghitalab, G. Baghersalimi, J. Text. Inst. 2017, 108, 1888.

[29] H. Zhang, Meas. Sci. Technol. 2015, 26, 105102.

[30] Y. Xiang, T. Li, Z. Suo, J. J. Vlassak, Appl. Phys. Lett. 2005, 87, 161910.

[31] J. Wang, C. Yan, K. Chee, P. Lee, Adv. Mater. 2015, 27, 2876. 


\section{WILEY-VCH}

[32] a) J. Y. Oh, M. Shin, J. B. Lee, J. H. Ahn, H. K. Baik, ACS Appl. Mater. Interfaces 2014, 6, 6954; b) S.-S. Yoon, D.-Y. Khang, J. Phys. Chem. C 2016, 120, 29525.

[33] a) A. M. Wessendorf, D. J. Newman, IEEE Trans. Biomed.Eng. 2012, 59, 3432. b) S.

Wagner, S. Lacour, J. Jones, P. Hsu, J. Sturm, T. Li, Z. Suo, Physica E 2004, 25, 326.

[34] M. Zahid, E. L. Papadopoulou, A. Athanassiou, I. S. Bayer, Mater. Des. 2017, 135, 213. 


\section{WILEY-VCH}
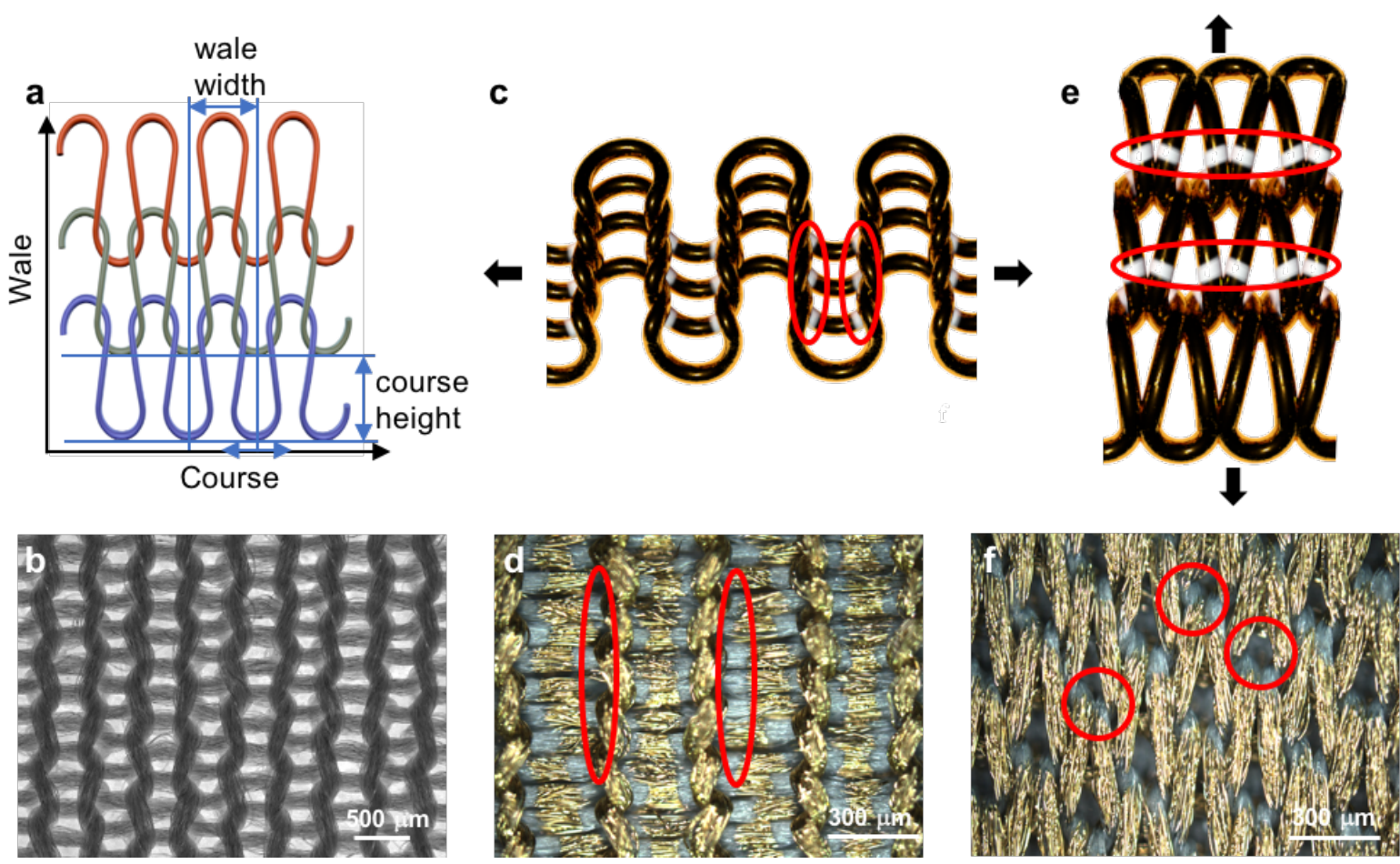

Figure 1. The weft-knitted textile structure. a) Schematic of the weft-knitted textile structure. b) Stereomicroscope image of the pristine textile. c-f) Illustrations and optical microscope images of PVD gold-coated textiles stretched in the (c, d) course direction and $(e, f)$ wale direction respectively. Red circles show examples of locations where gold deposition was blocked. 


\section{WILEY-VCH}


Figure 2. The weft-knitted textile coated with gold using ENIG. a) Stereomicroscope image of the gold-coated textile. Optical micrographs of the gold-coated textile b) stretched in the wale direction and c) in the course direction under 50\% strain. d,e) SEM images of d) pristine and e) gold-coated textile. f) Cross-sectional SEM image of the gold-coated textile. 


\section{WILEY-VCH}
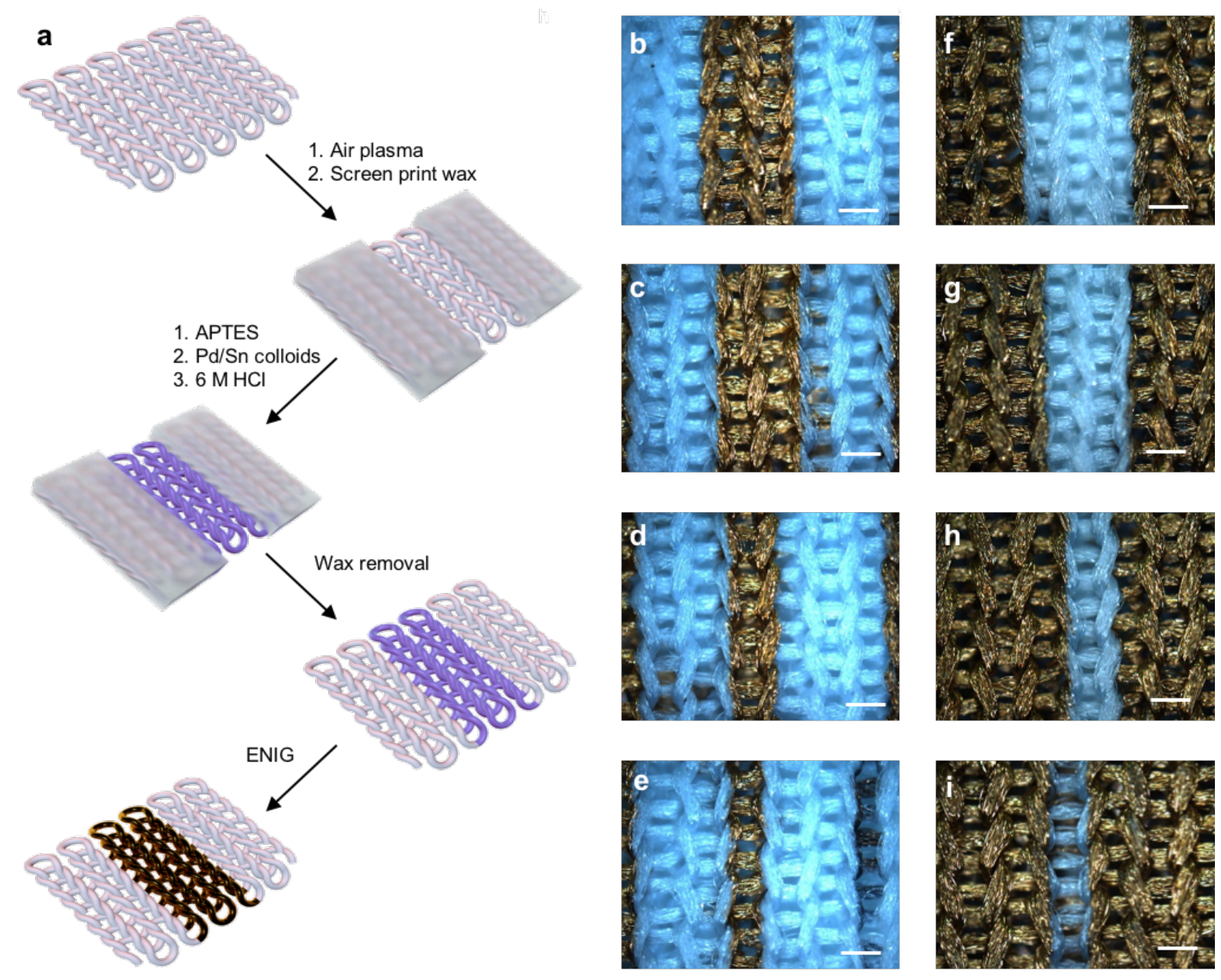

Figure 3. Patterning gold deposition on the knitted textile by wax screen printing. a) Schematic of the patterning process. b-e) Optical microscope images of gold lines with widths b) $1000 \mu \mathrm{m}$, c) $800 \mu \mathrm{m}$, d) $500 \mu \mathrm{m}$, e) $300 \mu \mathrm{m}$. f-i) Optical microscope images of linear gaps in gold with widths f) $1000 \mu \mathrm{m}, \mathrm{g}) 800 \mu \mathrm{m}, \mathrm{h}) 500 \mu \mathrm{m}$, i) $300 \mu \mathrm{m}$. Scale bar 400 $\mu \mathrm{m}$. 


\section{WILEY-VCH}

a

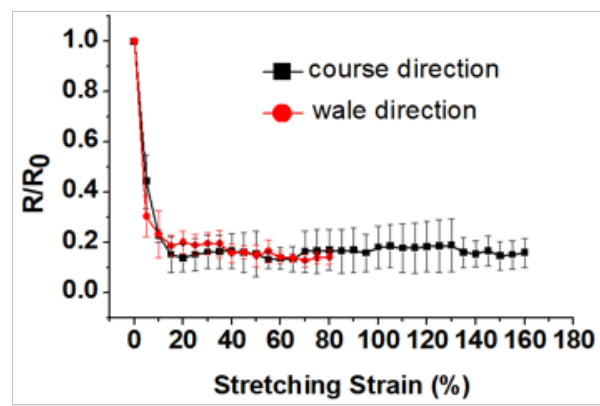

c

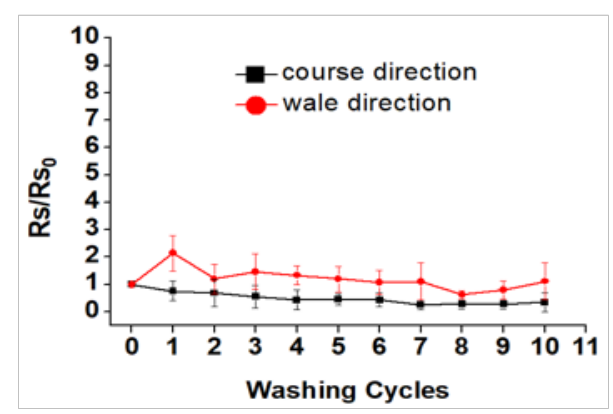

b

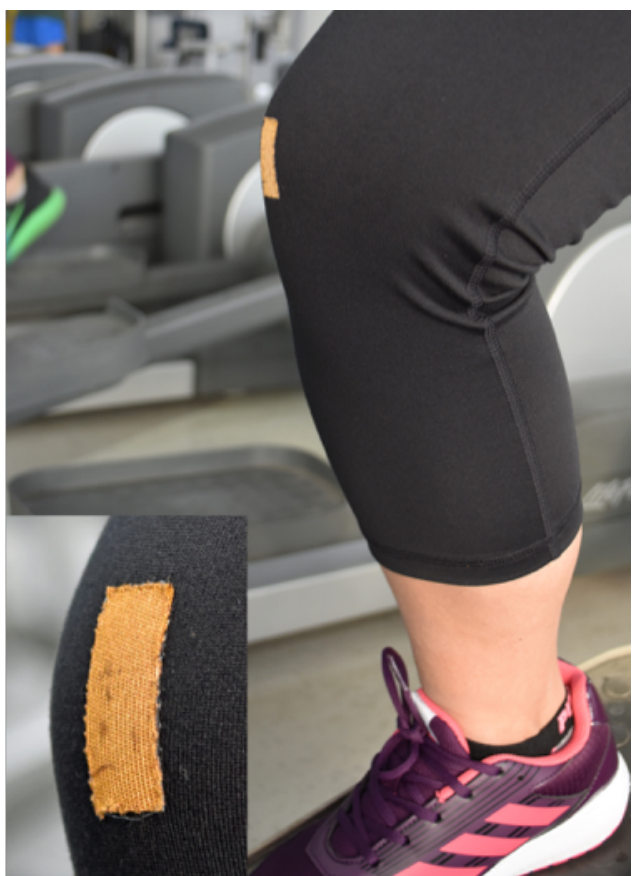

Figure 4. Stretchability, wearability, and durability of the gold-coated textile. a) Normalized change in resistance as a function of stretching strain along the course (black line) and wale (red line) direction. b) Photograph of gold-coated fabric sewn onto the knee section of exercise pants. c) Normalized change in sheet resistance after simulated laundry cycles. 


\section{WILEY-VCH}


Figure 5. Electroluminescent fabric. a) Schematic of electroluminescent fabric structure. b) Photographs of electroluminescent fabric at $0-40 \%$ strain. c, d) Wearable

electroluminescent fabric photographed alongside a retroreflective strip c) with and d) without an external light source. 


\section{WILEY-VCH}

Table of Contents Entry

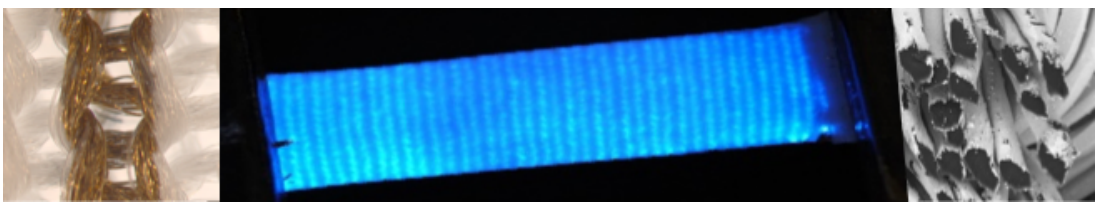

Y. Wu, S. S. Mechael, Y. Chen, Prof. T, B, Carmichael*

Solution Deposition of Conformal Gold Coatings on Knitted Fabric for E-Textiles and Electroluminescent Clothing

A solution-based method to coat the fibers of a knitted fabric with gold for stretchable etextiles is demonstrated. The metallization process preserves the textile structure, providing stretchable e-textiles that are highly conductive to $160 \%$ strain and durable to wearing, sweating, and washing. Patterned gold textiles are demonstrated by screen printing, and the fabrication of electroluminescent fabric intimately merges device function with textiles. 


\section{WILEY-VCH}

Copyright WILEY-VCH Verlag GmbH \& Co. KGaA, 69469 Weinheim, Germany, 2016.

\section{Supporting Information}

\section{Solution Deposition of Conformal Gold Coatings on Knitted Fabric for E-Textiles and Electroluminescent Clothing}

Yunyun Wu, Sara S. Mechael, Yiting Chen, and Tricia Breen Carmichael*

Characterization Optical microscopy was performed using an Olympus BX51 microscope equipped with an Olympus Q-Color3 digital camera. A micro-vice stretcher (S.T. Japan, USA, Inc.) was mounted to the microscope stage and samples were clamped in the stretcher to obtain microscope images of stretched samples. Stereomicrographs were taken using Leica m205 FA stereomicroscope with z-stack multi-plane focusing enabled. SEM images and energy dispersive X-ray spectroscopy were taken on an FEI Quanta 200 Environmental SEM. The energy dispersive X-ray spectroscopy (Figure S2) was recorded at 20 $\mathrm{kV}$.

XRD measurements were run on a PROTO AXRD powder diffractometer equipped with a $\mathrm{Cu}$ X-ray source, and a Mythen $1 \mathrm{~K}$ silicon strip detector, operated at $30 \mathrm{kV}$ and $20 \mathrm{~mA}$. Scans were performed using $\mathrm{CuK} \alpha$ radiation, a $0.2 \mathrm{~mm}$ divergence slit, and a step size of 0.02 degrees 2theta. X-ray diffraction patterns were obtained in the $2 \theta$ region from 30 to $140^{\circ}$. The XRD pattern for the gold-coated textile showed eight peaks at $38.3^{\circ}, 44.6^{\circ}, 64.8^{\circ}, 77.8^{\circ}, 82^{\circ}$, $98.8^{\circ}, 111^{\circ}, 115.5^{\circ}$, and $135.7^{\circ}$, corresponding to (111), (200), (220), (311), (222), (400), (331), (420), and (422) of fcc Au (JCPDF card 4-0784), respectively (Figure S2). JCPDF card 4-0784 for a gold standard has diffraction peaks at $38.184^{\circ}, 44.392^{\circ}, 64.576^{\circ}, 77.547^{\circ}, 81.721^{\circ}$, $98.133^{\circ}, 110.79^{\circ}, 115.25^{\circ}$, and $135.41^{\circ}$, corresponding to (111), (200), (220), (311), (222), (400), (331), (420), and (422). 


\section{WILEY-VCH}

MTS Criterion (model 43) was used for tensile testing under strain rate of $2.5 \mathrm{~mm} / \mathrm{s}$. For electrical characterization under strain, samples were clamped in the micro-vice stretcher and stretched manually in 5\% increments while measuring the resistance using a Keithley 2601A Sourcemeter. Textile-based light emitting device was operated at $165 \mathrm{~V}$ AC with a frequency of $37 \mathrm{kHz}$, which was inverted from 5 V DC using an inverter (PCU-554, TDK).

To measure bending strain at the knee, we marked two points on the knee and measured the distance between them in the straightened and bent position. We calculated the strain using percent difference between these values.


Figure S1. Photographs of a) face and b) back of gold-coated fabric.

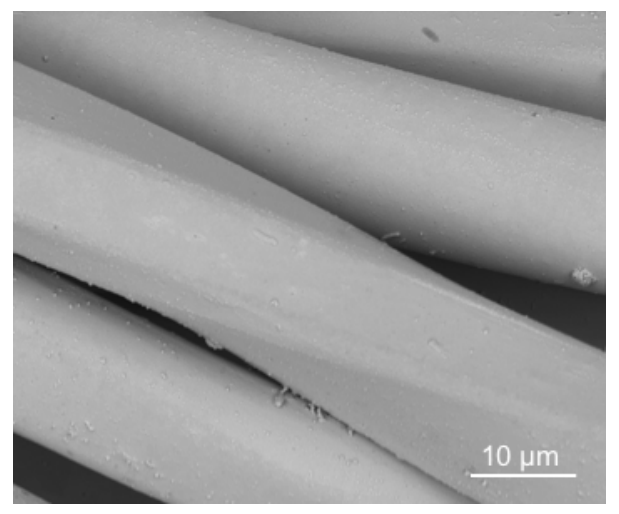

Figure S2. SEM image of the gold-coated textile. 


\section{WILEY-VCH}

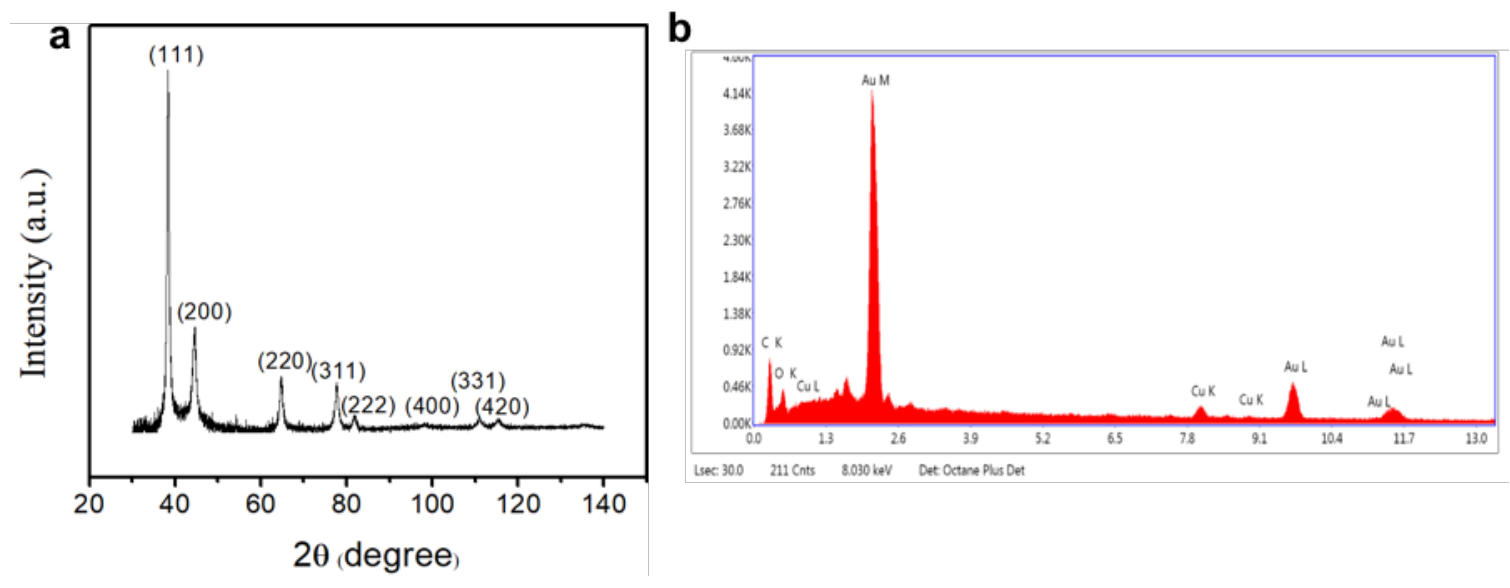

Figure S3. a) XRD patterns and b) EDX of gold-coated textiles.

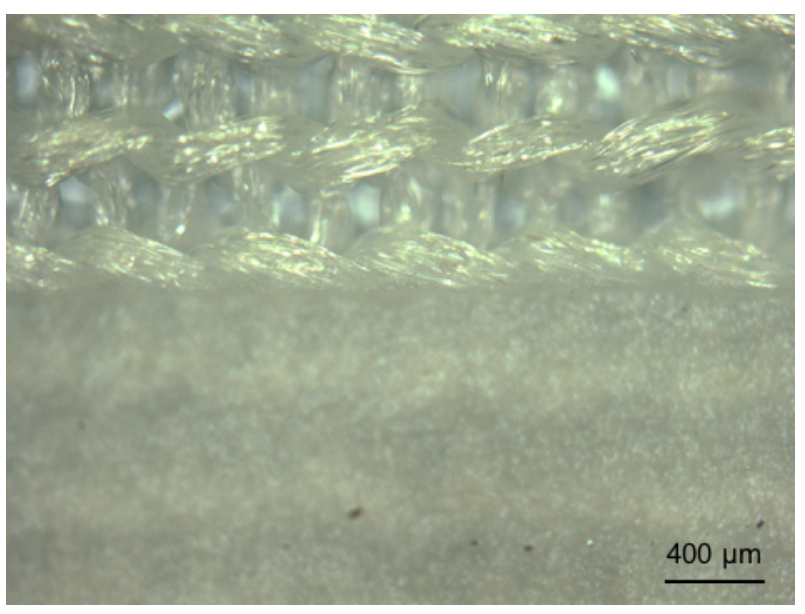

Figure S4. Optical micrograph of cold wax medium screen-printed on the knitted textile. 
a

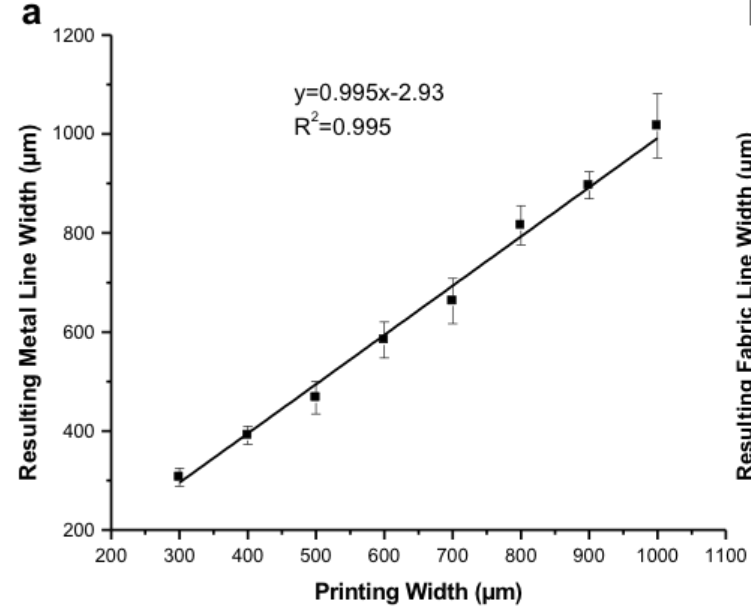

b

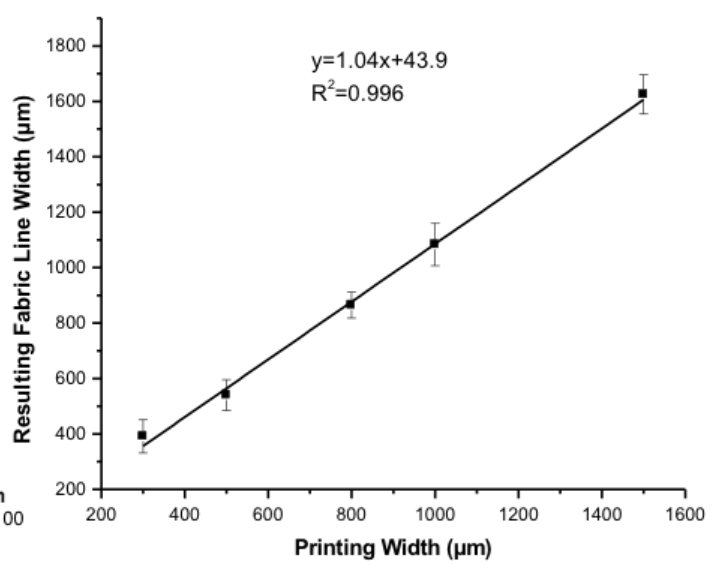

Figure S5. Plots of the width of the resulting a) gold lines and b) linear gaps in gold films as a function of the nominal line width of the stencil mask.
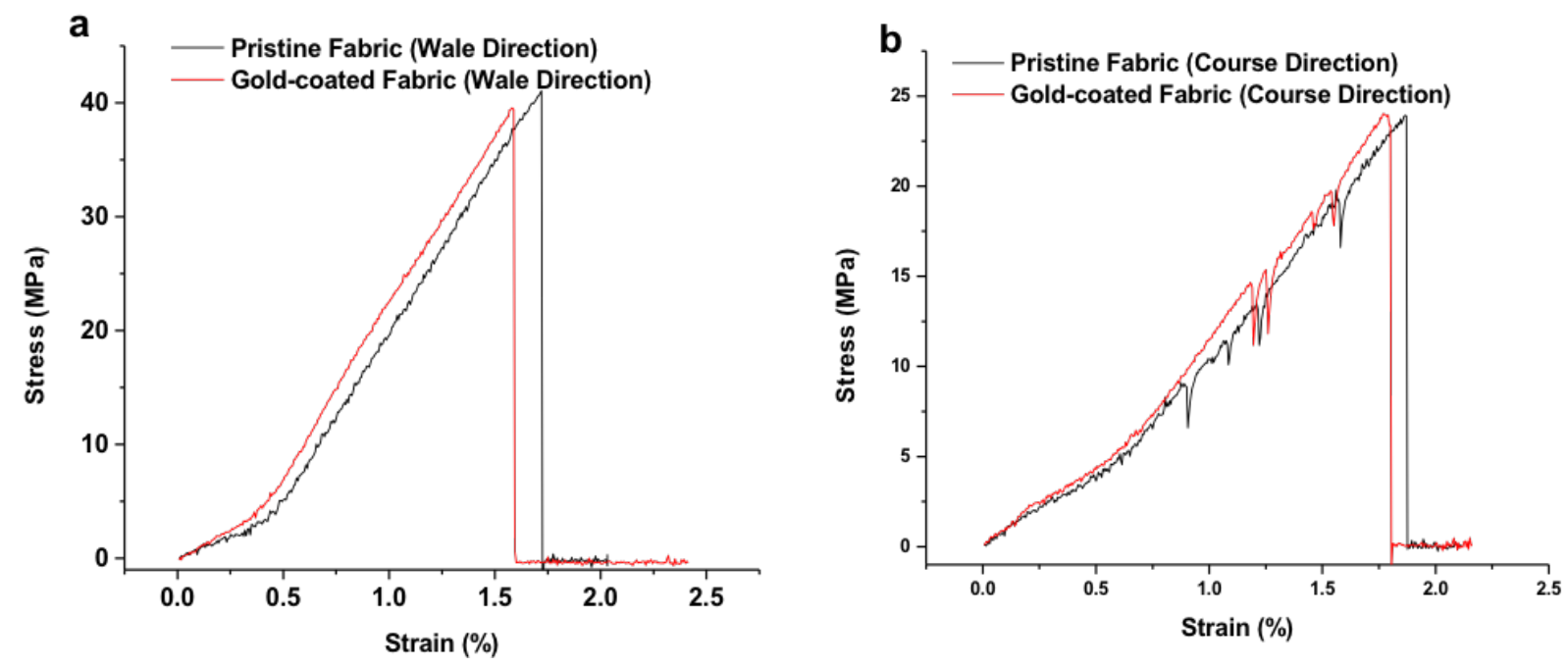

Figure S6. Stress strain curve of pristine fabric (black) and gold-coated fabric (red) in the a) wale direction and $b$ ) course direction. 


\section{WILEY-VCH}

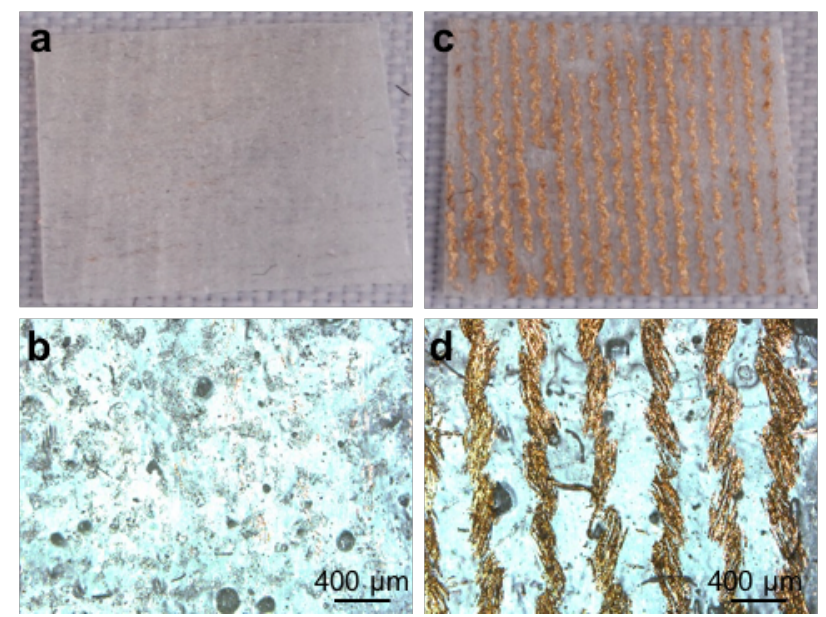

Figure S7. Photographs and optical microscope images of the tape after tape test of gold-coated textiles a, b) with APTES treatment and c, d) without APTES treatment.
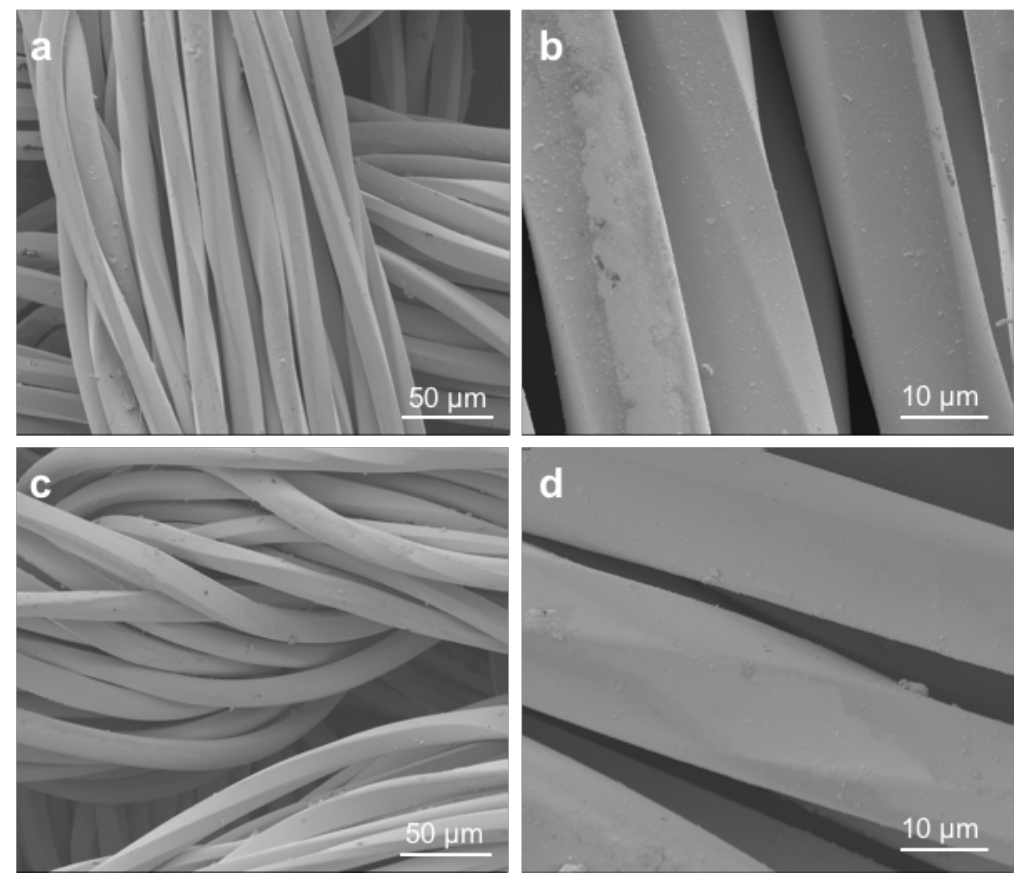

Figure S8. SEM of gold-coated textile at $20 \%$ strain $a, b$ ) in the wale direction and c,d) in the course direction. 


\section{WILEY-VCH}

a

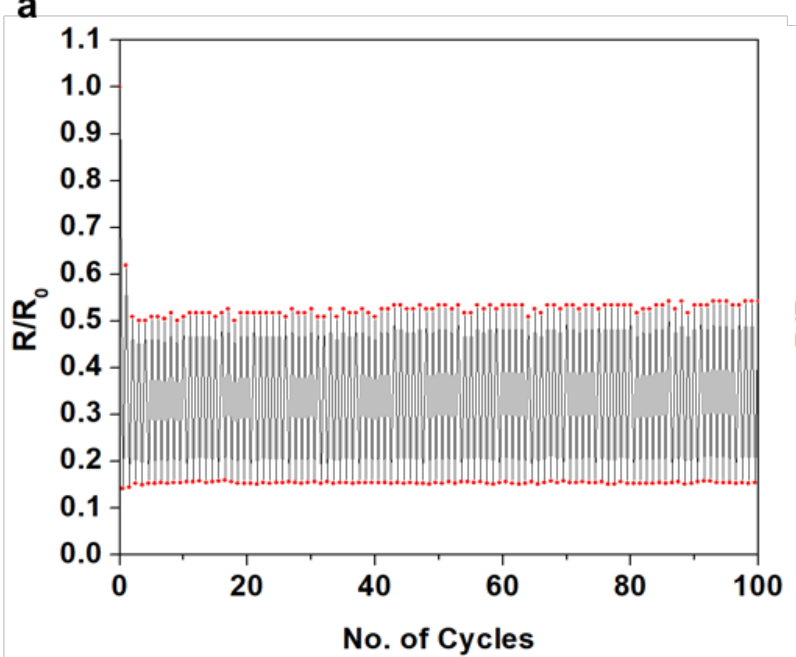

b



Figure S9. Normalized resistance of the gold-coated textile over 100 stretch-release cycles of $40 \%$ strain a) in the wale direction and b) in the course direction.

Table S1. Durability of gold-coated fabric to repetitive 40\% strain

\begin{tabular}{lcc} 
& $\begin{array}{c}\text { Left Leg } \\
\text { Resistance }^{\text {a) }} \\
{[\Omega]}\end{array}$ & $\begin{array}{c}\text { Right Leg } \\
\text { Resistance }^{\text {a) }} \\
{[\Omega]}\end{array}$ \\
\hline Before $5 \mathrm{~km}$ run & 9.3 & 10.1 \\
After $5 \mathrm{~km}$ run & 9.6 & 11.1 \\
\hline
\end{tabular}

${ }^{a)}$ Wale Direction 